\title{
SINR Balancing in the Downlink of Cognitive Radio Networks with Imperfect Channel Knowledge
}

\author{
Muhammad Fainan Hanif *, Peter J. Smith * and Mohamed-Slim Alouini ${ }^{\ddagger}$ \\ *Department of Electrical and Computer Engineering, University of Canterbury, Christchurch, New Zealand \\ ${ }^{\ddagger}$ Electrical Engineering Program, KAUST, Thuwal, Saudi Arabia \\ Email:mfh21@uclive.ac.nz,p.smith@elec.canterbury.ac.nz,slim.alouini@kaust.edu.sa
}

\begin{abstract}
In this paper we consider the problem of signal-tointerference-plus-noise ratio (SINR) balancing in the downlink of cognitive radio (CR) networks while simultaneously keeping interference levels at primary user (PU) receivers (RXs) below an acceptable threshold with uncertain channel state information available at the CR base-station (BS). We optimize the beamforming vectors at the CR BS so that the worst user SINR is maximized and transmit power constraints at the CR BS and interference constraints at the PU RXs are satisfied. With uncertainties in the channel bounded by a Euclidean ball, the semidefinite program (SDP) modeling the balancing problem is solved using the recently developed convex iteration technique without relaxing the rank constraints. Numerical simulations are conducted to show the effectiveness of the proposed technique in comparison to known approximations.
\end{abstract}

\section{INTRODUCTION}

The problem of maximizing the worst signal-tointerference-plus-noise-ratio (SINR) of a cognitive radio (CR) device in the downlink of a CR network, operating in the vicinity of multiple primary users (PUs) was solved in [1] by obtaining optimal beamforming vectors using the uplink-downlink duality concept. The work of [1] was recently extended to the case of maximizing the worst user SINR with imperfect channel estimates at the CR base station (BS) [2]. This extension assumed that errors in the channel state information (CSI) are bounded by ellipsoids. Based on this channel matrix uncertainty model, the problem was formulated as a quasi-convex optimization program using the $\mathcal{S}$-procedure [3]. To be precise, in the case of channel uncertainty, the authors first derived an equivalent problem that involved rank-1 constraints on the positive semidefinite (PSD) matrices modeling the beamforming vectors. These constraints rendered the problem non-convex. Later, the problem was solved by relaxing the rank-1 constraints and using the bisection search [4] algorithm.

Relaxing such rank constraints results in an upper bound to the optimal solution. However, under certain conditions (e.g., by showing that the optimum cost of original problem is equal to that of the Lagrange dual of its relaxed version [5]) we can obtain the optimal solution, one with rank-1 constraints satisfied. In addition to this, there are many optimization problems in communications, for example [6]-[8] to name just a few, that can be formulated as semidefinite programs (SDPs) [4] with rank constraints. As a result of these nonconvex rank constraints various relaxations and approximations are usually adopted. Different techniques have also been developed to obtain approximate rank-1 solutions [7], [8] from the optimized, but relaxed, variables. In [9] the problem of rank constrained SDPs modeling power optimization in the downlink of CR networks with perfect channel estimates available at the BS is studied. By elegantly fine tuning the results of [10], SDP relaxation of three classes of optimal beamforming problem were shown to always possess rank-1 solutions in [9].

In this paper, we study the problem of rank constrained SINR balancing in the downlink of CR networks with imperfect channel estimates available at the CR BS. In particular, our approach does not require any relaxation of rank constraints. Our main contributions include showing successful incorporation of the recently developed convex iteration [11] technique and developing a low complexity algorithm to solve the problem.

The remaining paper is organized as follows: In Section II we describe the system model. The analytical framework is presented in Section III and in Section IV we detail our proposed solution. Finally, the results and conclusions are presented in Sections V and VI respectively.

Throughout the paper matrices are represented using boldface uppercase letters while boldface lowercase letters are used for vectors. $\operatorname{Tr}(),.(.)^{\dagger}$ and $\mathbb{E}($.$) denote the trace, the conjugate$ transpose and statistical expectation operators respectively. I denotes an identity matrix of appropriate size. $\mathbb{C}^{x \times y}$ denotes the space of $x \times y$ matrices with complex entries. $\mathcal{C N}(0, \Gamma)$ represents the distribution of a zero mean circularly symmetric complex Gaussian random variable with variance $\Gamma .\|$.$\| is the$ $l_{2}$ norm operator. $\mathbf{M}(:, j)$ is used to represent the $j$ th column of a matrix M. $[i: l]$ gives a row vector from $i$ to $l$ with unit increments in the entries. $\mathbf{M} \succeq \mathbf{0}$ indicates that $\mathbf{M}$ is a PSD matrix. $\mathbb{S}^{N}, \mathbb{R}^{N}$ and $\mathbb{R}$ represent subspaces of the set of $N \times N$ Hermitian-symmetric matrices, the set of real $N$-vectors and the set of real numbers, respectively. $\operatorname{diag}(\mathbf{N})$ returns the principal diagonal of a square matrix $\mathbf{N}$ and $\mathbf{M} \succeq \mathbf{I}$ indicates that $\mathbf{M}-\mathbf{I} \succeq \mathbf{0}$ is PSD.

\section{SySTEM MODEL}

Let us consider a CR BS having $N_{C R}$ antennas serving $C$ single antenna cognitive devices. It is assumed that the cognitive broadcast channel is operating in the vicinity of $K$ single antenna PU receivers (RXs). Suppose $\mathrm{s} \in \mathbb{C}^{C}$ represents the column vector of data symbols meant for the 
$C$ different CR RXs such that the symbol for the $i$ th $\mathrm{RX}$ is $s_{i} \sim \mathcal{C N}(0,1), i=1, \ldots, C$. The CR BS transmits a vector, $\mathbf{x} \in \mathbb{C}^{N_{C R}}$, obtained by linear precoding of $\mathbf{s}$, i.e.,

$$
\mathbf{x}=\sum_{j=1}^{C} \mathbf{p}_{j} s_{j}=\mathbf{P} \mathbf{s}
$$

where $\mathbf{P} \in \mathbb{C}^{N_{C R} \times C}$ is the precoding matrix and $\mathbf{p}_{j}$ represents its $j$ th column. With the above model we have $\mathbb{E}\left(\mathbf{s s}^{\dagger}\right)=$ I. Thus, assuming that the total available power is $P_{\text {total }}$, the constraint on the transmit power becomes $\mathbb{E}\left(\|\mathbf{x}\|^{2}\right)=$ $\sum_{j=1}^{C}\left\|\mathbf{p}_{j}\right\|^{2}=\operatorname{Tr}\left(\mathbf{P}^{\dagger} \mathbf{P}\right) \leq P_{\text {total }}$. The BS then transmits the vector $\mathrm{x}$ over a quasi-static flat-fading channel. The received signal, $y_{i}$, at the $i$ th CR user is given by:

$$
y_{i}=\mathbf{h}_{i} \mathbf{x}+n_{i}=\mathbf{h}_{i} \mathbf{p}_{i} s_{i}+\sum_{\substack{j=1 \\ j \neq i}}^{C} \mathbf{h}_{i} \mathbf{p}_{j} s_{j}+n_{i}
$$

where $\mathbf{h}_{i} \in \mathbb{C}^{1 \times N_{C R}}$ is the downlink channel vector for the $i$ th user and $n_{i} \sim \mathcal{C N}\left(0, \sigma_{i}^{2}\right)$. In a similar way, the signal received at the $m$ th $\mathrm{PU} \mathrm{RX}, z_{m}$ is:

$$
z_{m}=\mathbf{h}_{\text {int }_{m}} \mathbf{x}+v_{m}=\sum_{n=1}^{C} \mathbf{h}_{\text {int }_{m}} \mathbf{p}_{n} s_{n}+v_{m},
$$

where $\mathbf{h}_{\text {int }_{m}} \in \mathbb{C}^{1 \times N_{C R}}$ and $v_{m} \sim \mathcal{C N}\left(0, \sigma_{m}^{2}\right)$ are the channel and noise at the $m$ th PU RX, respectively.

Channel Uncertainty: In practice the CR BS is not able to obtain perfect CSI for both the CR-CR and CR-PU channels. We model the CSI error for the CR-CR channel $\mathbf{h}_{i}$ as:

$$
\mathbf{h}_{i}=\hat{\mathbf{h}}_{i}+\mathbf{e}_{i}
$$

where $\hat{\mathbf{h}}_{i}$ is the estimated channel for user $i$ at the CR BS and $\mathbf{e}_{i} \in \mathcal{H}_{i}\left(\delta_{i}\right)$ where

$$
\mathcal{H}_{i}\left(\delta_{i}\right)=\left\{\mathbf{a}_{i}:\left\|\mathbf{a}_{i}\right\| \leq \delta_{i}\right\} .
$$

Similarly, the channels for the CR-PU links are modeled as

$$
\mathbf{h}_{\text {int }_{m}}=\hat{\mathbf{h}}_{\text {int }_{m}}+\mathbf{e}_{\text {int }_{m}},
$$

where $\hat{\mathbf{h}}_{\text {int }_{m}}$ is the estimated channel for the $m$ th PU at the CR BS and $\mathbf{e}_{\text {int }_{m}} \in \mathcal{H}_{\text {int }_{m}}\left(\delta_{\text {int }_{m}}\right)$ where

$$
\mathcal{H}_{\text {int }_{m}}\left(\delta_{\text {int }_{m}}\right)=\left\{\mathbf{b}_{m}:\left\|\mathbf{b}_{m}\right\| \leq \delta_{\text {int }_{m}}\right\} .
$$

The ball uncertainty models described above are common models used to characterize errors caused by processes such as quantization and estimation [12].

\section{ANALYTICAL FRAMEWORK}

With the system model elaborated in Sec. II, it is easy to see that the total signal power received at the $i$ th CR RX, $P_{i} \triangleq \mathbb{E}\left(\left|y_{i}\right|^{2}\right)$, is given by:

$$
P_{i}=\mathbf{h}_{i} \mathbf{p}_{i} \mathbf{p}_{i}^{\dagger} \mathbf{h}_{i}^{\dagger}+\sum_{\substack{j=1 \\ j \neq i}}^{C} \mathbf{h}_{i} \mathbf{p}_{j} \mathbf{p}_{j}^{\dagger} \mathbf{h}_{i}^{\dagger}+\sigma_{i}^{2}
$$

where the first term on the right hand side of (8) is the power of the desired signal while the second term represents the inter- user interference. Thus, for user $i$, the SINR is defined by:

$$
\operatorname{SINR}_{i}=\frac{\left(\hat{\mathbf{h}}_{i}+\mathbf{e}_{i}\right) \mathbf{p}_{i} \mathbf{p}_{i}^{\dagger}\left(\hat{\mathbf{h}}_{i}+\mathbf{e}_{i}\right)^{\dagger}}{\sum_{\substack{j=1 \\ j \neq i}}^{C}\left(\hat{\mathbf{h}}_{i}+\mathbf{e}_{i}\right) \mathbf{p}_{j} \mathbf{p}_{j}^{\dagger}\left(\hat{\mathbf{h}}_{i}+\mathbf{e}_{i}\right)^{\dagger}+\sigma_{i}^{2}}, \quad i=1, \ldots, C .
$$

Similarly, the interference power at the $m$ th $\mathrm{PU}, \mathrm{IP}_{m}$, is:

$\mathrm{IP}_{m}=\sum_{n=1}^{C}\left(\hat{\mathbf{h}}_{\mathrm{int}_{m}}+\mathbf{e}_{\text {int }_{m}}\right) \mathbf{p}_{n} \mathbf{p}_{n}^{\dagger}\left(\hat{\mathbf{h}}_{\text {int }_{m}}+\mathbf{e}_{\text {int }_{m}}\right)^{\dagger}, \quad m=1, \ldots K$.

\section{A. Problem Formulation}

We aim to maximize the minimum SINR of the users in the CR system subject to transmit power constraints while simultaneously maintaining interference power at the PU RXs below a certain acceptable threshold. These problems, in the context of ordinary broadcast channels, are generally categorized as max-min fair SINR problems [12], [13]. However, we perform the maximization of the weakest SINR over the channel uncertainty models described above. Thus, we solve:

$$
\begin{aligned}
\mathcal{P}_{1}: \underset{t, \mathbf{P}}{\operatorname{maximize}} & t \\
\text { subject to } & \operatorname{SINR}_{i} \geq t, i=1, \ldots, C, \forall \mathbf{e}_{i} \in \mathcal{H}_{i}\left(\delta_{i}\right) \\
& \operatorname{IP}_{m} \leq \zeta_{m}, \forall \mathbf{e}_{\text {int }_{m}} \in \mathcal{H}_{\text {int }_{m}}\left(\delta_{\text {int }_{m}}\right) \\
& \sum_{n=1}^{C} \operatorname{Tr}\left(\mathbf{p}_{i} \mathbf{p}_{i}^{\dagger}\right) \leq P_{\text {total }}
\end{aligned}
$$

where $\zeta_{m}$ is the maximum tolerable interference level for the $m$ th PU RX.

\section{B. Approximate Solution of $\mathcal{P}_{1}$}

Recently, $\mathcal{P}_{1}$ has been solved in [2] by using the powerful $\mathcal{S}$-procedure [3]. Their result for the case of spherical channel uncertainty can be written as:

Lemma 1 ( [2]): With the substitution, $\mathbf{W}_{i}=\mathbf{p}_{i} \mathbf{p}_{i}^{\dagger}$, problem $\mathcal{P}_{1}$ can be equivalently rewritten as:

$$
\begin{aligned}
& \mathcal{P}_{2} \text { : maximize } t \\
& { }_{n}, t, \mathbf{W}_{i} \\
& \text { subject to }\left[\begin{array}{rr}
\hat{\mathbf{h}}_{i} \mathbf{Q}_{i} \hat{\mathbf{h}}_{i}^{\dagger}-t \sigma^{2}-\tau_{i} \delta_{i}^{2} & \hat{\mathbf{h}}_{i} \mathbf{Q}_{i} \\
\left(\hat{\mathbf{h}}_{i} \mathbf{Q}_{i}\right)^{\dagger} & \mathbf{Q}_{i}+\tau_{i} \mathbf{I}
\end{array}\right] \succeq 0 \\
& \tau_{i} \geq 0, i=1,2, \ldots, C \\
& {\left[\begin{array}{cc}
-\hat{\mathbf{h}}_{\text {int }_{m}} \mathbf{S}_{\hat{\mathbf{h}}_{\text {int }_{m}}^{\dagger}}^{\dagger}+\zeta_{m}-\lambda_{m} \delta_{\text {int }_{m}}^{2} & -\hat{\mathbf{h}}_{\text {int }_{m}} \mathbf{S} \\
\left(-\hat{\mathbf{h}}_{\text {int }_{m}} \mathbf{S}\right)^{\dagger} & -\mathbf{S}+\lambda_{m} \mathbf{I}
\end{array}\right] \succeq 0} \\
& \lambda_{m} \geq 0, m=1,2, \ldots, K \\
& \operatorname{Tr}(\mathbf{S}) \leq P_{\text {total }}, \mathbf{W}_{i} \succeq 0, \operatorname{rank}\left(\mathbf{W}_{i}\right)=1, \forall i .
\end{aligned}
$$

where $\mathbf{Q}_{i}=\mathbf{W}_{i}-t \sum_{\substack{j \neq 1 \\ j \neq i}}^{C} \mathbf{W}_{j}$ and $\mathbf{S}=\sum_{i=1}^{C} \mathbf{W}_{i}$.

Clearly $\mathcal{P}_{2}$ is non-convex owing to the rank-1 constraints. By relaxing the rank-1 constraints, the problem becomes a quasiconvex optimization problem and hence can be efficiently solved using the one dimensional bisection search algorithm [4], [12], [14]. In [2], the authors modify the basic bisection search by storing those iterations that returned rank-1 solutions and later, if the final beamforming solution is not optimal, they use that rank-1 solution which produced the largest SINR. It is 
worth noting that the above solution does not incorporate the effect of interference from PU TXs to CR RXs. Due to space constraints we do not explicitly present results including this effect and leave the details to the forthcoming journal version. However, preliminary results are shown in Sec. V.

\section{PRoposed Solution}

As is evident from the above discussion, the known solution to the problem at hand is approximate due to the rank-1 constraints. There are many problems in communications [6][8] that involve such constraints on the rank of unknown matrix variables and these are often solved based on some approximating technique. Motivated by this we attempt to present a solution to $\mathcal{P}_{2}$ without relaxing the rank constraints. We replace the feasibility problem associated with $\mathcal{P}_{2}$ with a set of equivalent convex programs that are solved iteratively in conjunction with the bisection search to obtain rank-1 solutions. Our technique utilizes convex iteration algorithm [11] approach to solve rank constrained SDPs.

\section{A. The Main Idea of "Convex Iteration" [11]}

Suppose we are given a SDP in the variable $\mathbf{F} \in \mathbb{S}^{N}$ and we are to solve the following corresponding semidefinite feasibility problem:

$$
\begin{aligned}
\mathcal{F P}: \text { find } & \mathbf{F} \\
\text { subject to } & \mathbf{F} \in \mathcal{C}, \mathbf{F} \succeq 0, \operatorname{rank}(\mathbf{F}) \leq n
\end{aligned}
$$

where $\mathcal{C}$ is a convex set assumed to contain PSD matrices with rank- $n$ or less. Then, $\mathbf{F}$ can be determined by iteratively solving the following two convex problems:

$$
\begin{aligned}
\mathcal{F P}_{1}: \underset{\mathbf{F}}{\operatorname{minimize}} & \operatorname{Tr}(\mathbf{F D}) \\
\text { subject to } & \mathbf{F} \in \mathcal{C}, \mathbf{F} \succeq 0
\end{aligned}
$$

where $\mathbf{D} \in \mathbb{S}^{N}$ is the direction vector obtained by solving the following SDP,

$$
\begin{aligned}
\mathcal{F P}_{2}: \underset{\mathbf{D}}{\operatorname{minimize}} & \operatorname{Tr}\left(\mathbf{F}^{\star} \mathbf{D}\right) \\
\text { subject to } & \mathbf{0} \preceq \mathbf{D} \preceq \mathbf{I}, \operatorname{Tr}(\mathbf{D})=N-n
\end{aligned}
$$

where $\mathbf{F}^{\star}$ is an optimal solution to $\mathcal{F} \mathcal{P}_{1}$ for some given iterate $\mathrm{D}$. We continue iterating between $\mathcal{F} \mathcal{P}_{1}$ and $\mathcal{F} \mathcal{P}_{2}$ until $\operatorname{Tr}\left(\mathbf{F}^{\star} \mathbf{D}^{\star}\right)$ attains a vanishingly small value, where $\mathbf{D}^{\star}$ is the optimal direction vector. After convergence is achieved the final pair $\left(\mathbf{D}^{\star}, \mathbf{F}^{\star}\right)$ provides an $\mathbf{F}^{\star}$ with $\operatorname{rank}\left(\mathbf{F}^{\star}\right) \leq n$, if it existed in $\mathcal{C}$. We stress that iteratively solving $\mathcal{F} \mathcal{P}_{1}$ and $\mathcal{F} \mathcal{P}_{2}$ gives an equivalent representation [4] of the original problem $\mathcal{F} \mathcal{P}$ instead of a relaxation and a solution of rank- $n$ or less is obtained if it exists in $\mathcal{C}$.

\section{B. Incorporation of "Convex Iteration" in $\mathcal{P}_{2}$}

As mentioned above, the relaxed version of $\mathcal{P}_{2}$ can be efficiently solved by performing a bisection search. Such a search involves solving a convex semidefinite feasibility program for a particular value of $t$ and then, depending on whether or not the program is feasible for that $t$, the search interval for the next step is updated accordingly [4].

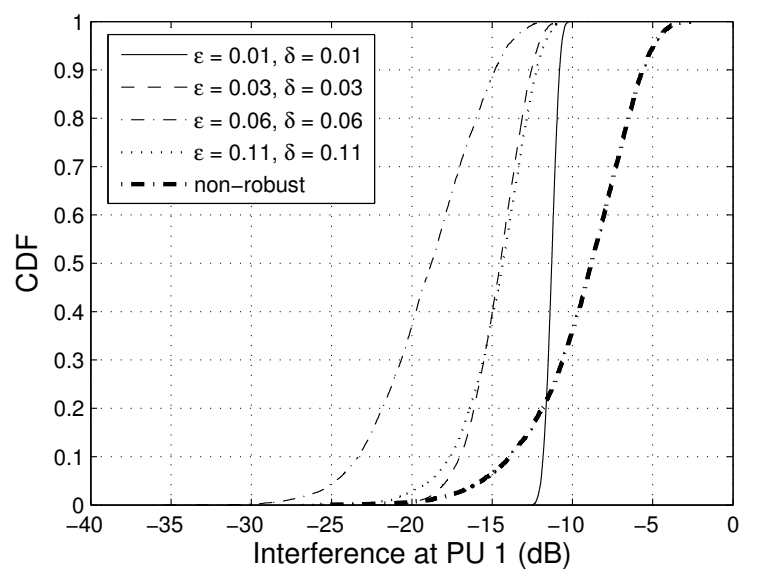

Fig. 1. CDF of the interference due to the CR BS at the first PU for different values of $\epsilon, \delta$.

In contrast, we execute the convex iteration described above together with the bisection search. This avoids the need for any relaxation. This modified procedure is detailed in Algorithm 1. $I / P, O / p$ represent input and output of the algorithm respectively, comments are indicated by the symbol ' $D$ ', while the symbol ' $\star$ ' represents the optimum value. The Boolean condition true for the inner while loop indicates that the loop keeps on executing until we break out of it. $k$ denotes the iteration index and $\mathbf{W}^{(k)}, t^{(k)}$ represent the values of $\mathbf{W}$ and $t$ in the $k$ th iteration, respectively. The sets $\mathcal{H}_{W}$ and $\mathcal{H}_{t}$ are used to collect the values of $\mathbf{W}$ and $t$, respectively, for each iteration. For implementation purposes the set $\mathcal{H}_{W}$ can be updated with special matrices (for example, zero matrices) when the problem is found to be infeasible. Furthermore, in Algorithm 1, SINRmax and SINRmin represent the upper and lower limits on the SINR of the system and we iterate the bisection search until the difference between these limits is less than or equal to some threshold, 'thresh.1'. To solve the rank constrained problem exactly, we replace the convex feasibility problem for some iterate, $t$, with the equivalent convex iteration. This is shown in lines 9-12 of Algorithm 1. Similarly, the second convex program giving the optimal direction vectors is included in line 16 of the algorithm. Note that we terminate the convex iteration when either we have obtained all precoders of desired rank (line 17) or once we have determined that the value of the objective has become sufficiently small i.e., smaller than some threshold, 'thresh.2' (line 20) to deduce that no such rank matrices are available. We remark that the precoding matrices and the corresponding $t_{0}$ can be obtained from $\mathcal{H}_{W}$ and $\mathcal{H}_{t}$ respectively, by observing the last entries of these sets for which the problem was found to be feasible. Algorithm 1 does not contain any procedure for handling stalls as these were not observed in the simulations. Various computational issues related to the convex iteration approach are presented in the expanded journal version.

\section{RESUlts}

In order to validate the proposed scheme, numerical simulations are performed. To conduct these simulations it is assumed that the CR BS is equipped with a uniform linear 


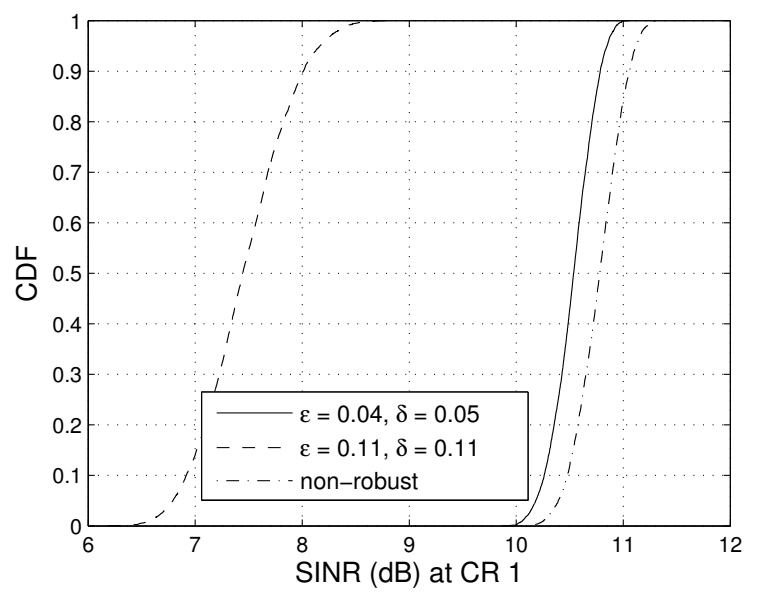

Fig. 2. CDF of SINR at the first CR RX for different values of $\epsilon, \delta$ without interference from the PU TXs.

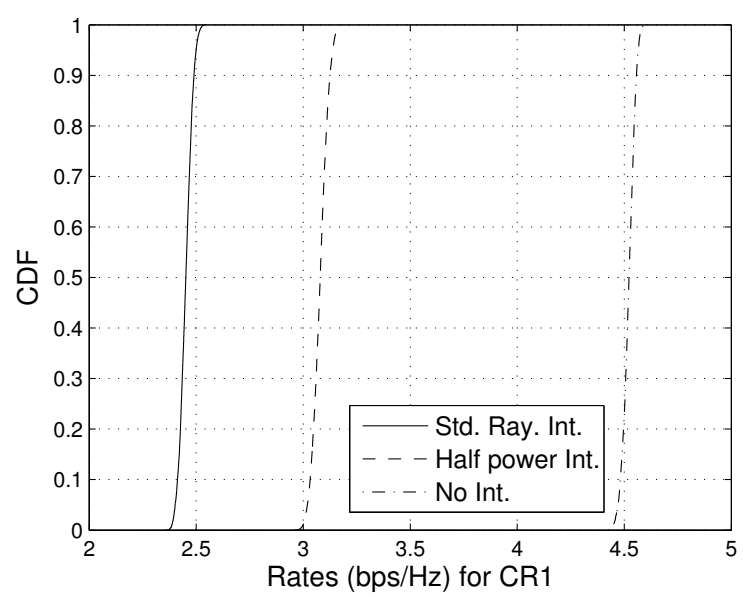

Fig. 3. Effect of licensed user interference on the rates of the first CR device. The interfering PU signals are Rayleigh faded.

array having $N_{C R}=5$ elements spaced half a wavelength apart. Further, the BS serves $3 \mathrm{CR}$ devices located at angles $\theta_{1}=20^{\circ}, \theta_{2}=45^{\circ}$ and $\theta_{3}=60^{\circ}$ with respect to antenna broadside, respectively. Similarly, 2 PU devices are assumed to be located along the directions of $\phi_{1}=40^{\circ}$ and $\phi_{2}=75^{\circ}$ respectively. At the CR receivers the noise is taken as $\mathcal{C N}(0,1)$. Unless otherwise stated, the maximum tolerable interference-to-noise ratio level at the PU RXs is assumed to be $\zeta_{m}=\zeta=-10 \mathrm{~dB}$. With these assumptions, the channels from the BS to the CR and PU RXs, for $o=1 \ldots N_{C R}$, are respectively given by the following simple model [15]:

$$
\begin{gathered}
\left(\hat{\mathbf{h}}_{i}\right)_{o}=e^{\frac{j 2 \pi d}{\lambda}(o-1) \cos \left(\theta_{i}\right)}, i=1,2,3, \\
\left(\hat{\mathbf{h}}_{\text {int }_{m}}\right)_{o}=e^{\frac{j 2 \pi d}{\lambda}(o-1) \cos \left(\phi_{m}\right)}, m=1,2 .
\end{gathered}
$$

where $d$ and $\lambda$ represent antenna separation and wavelength (we have taken $d / \lambda=0.5$ ) respectively and $j=\sqrt{-1}$. For each true channel realization, the corresponding CSI error vector is normally distributed, truncated to lie within a sphere of radius $\delta_{i}=\delta, \forall i$ and $\delta_{\text {int }_{m}}=\epsilon, \forall m$ for the CR and PU channels respectively. While implementing Algorithm 1 'thresh.1' and 'thresh.2' are taken as 0.05 and $1 \mathrm{e}-10$ respectively. In addition to this, it is assumed that SINRmin $=0 \mathrm{~dB}$, SINRmax $=13.01 \mathrm{~dB}$ and the average total transmit signal-to-noise ratio (SNR) is $P_{\text {total }} / N_{0}=13.97 \mathrm{~dB}$. To make a comparison, results for the non-robust case have also been plotted. In the non-robust case, the beamformers are obtained based on the estimated channels by ignoring the uncertainty regions. The optimization problems (SDPs) are solved using CVX [16]. First of all we compare the ranks of the beamforming matrices obtained using the proposed method and the ranks of the beamforming matrices obtained using the algorithm presented in [2]. To do so, we implement [2, Eqs. (16a) and (16b)] in [2, Algorithm 1]. Furthermore, [2, Algorithm 1] is slightly modified by noting the rank of the final solution only. With the implementation details outlined in the expanded journal version of this work, it is observed that Algorithm 1 in [2] always returns full rank matrices in the final iteration for all values of $\epsilon$ and $\delta$ considered. In contrast, unit rank beamforming matrices are obtained using the proposed method.

In a second experiment the cumulative distribution function (CDF) of interference at the first PU RX is obtained for different values of $\epsilon$ and $\delta$ (see Fig. 1). The CDF is taken over the distribution of possible channels which lie in the ball uncertainty regions for the given channel estimates. Results for the case of a non-robust design are also plotted. It is clear from Fig. 1 that, as expected, the non-robust design performs the worst. In fact, it exceeds the interference threshold $(-10$ $\mathrm{dB}$ ) around $64 \%$ of the time. Furthermore, the interference in Fig. 1 shows an interesting trend. For small values of $\epsilon, \delta$ the CR TX is able to direct a sharp null in its beam pattern towards each PU RX as the channel is precisely known. As $\epsilon, \delta$ are increased, at first the CDFs spread out since the range of possible channels is the uncertainty ball increases and most of these channels lie in a deep null. As $\epsilon, \delta$ are increased further the channel knowledge decreases and the TX has to use a broad null which is less deep to cater for a wide range of possible channels. Hence, the beam pattern flattens out in the direction of the uncertainty region and the interference values become less variable. This is shown in Fig. 1 where the spread of the CDF increases from $\epsilon=\delta=0.01$ to $\epsilon=\delta=0.06$ but then decreases for $\epsilon=\delta=0.11$. This observation has another implication. For small $\epsilon, \delta$ the sharp nulls mean that the interference constraint can be attained by beamforming and then full transmit power can be used to boost the CR-CR SINR. However, for larger $\epsilon, \delta$ values the beam pattern does not have sharp nulls and power back-off may be required. For example, in Fig. 1 full transmit power was used for $\epsilon=\delta \in\{0.01,0.03,0.06\}$ but a $20 \%$ back off was required for $\epsilon=\delta=0.11$.

In Fig. 2 the CDF for the SINR at CR RX 1 for different values of $\epsilon, \delta$ is plotted. As in Fig. 1, the CDF is over the possible channels in the uncertainty region. It is seen that, as expected, higher $\epsilon, \delta$ results in a decrease in the mean SINR as decreased channel knowledge limits the ability of the TX to direct strong beams towards the CR RXs while simultaneously directing nulls towards the PU RXs. The non-robust design 


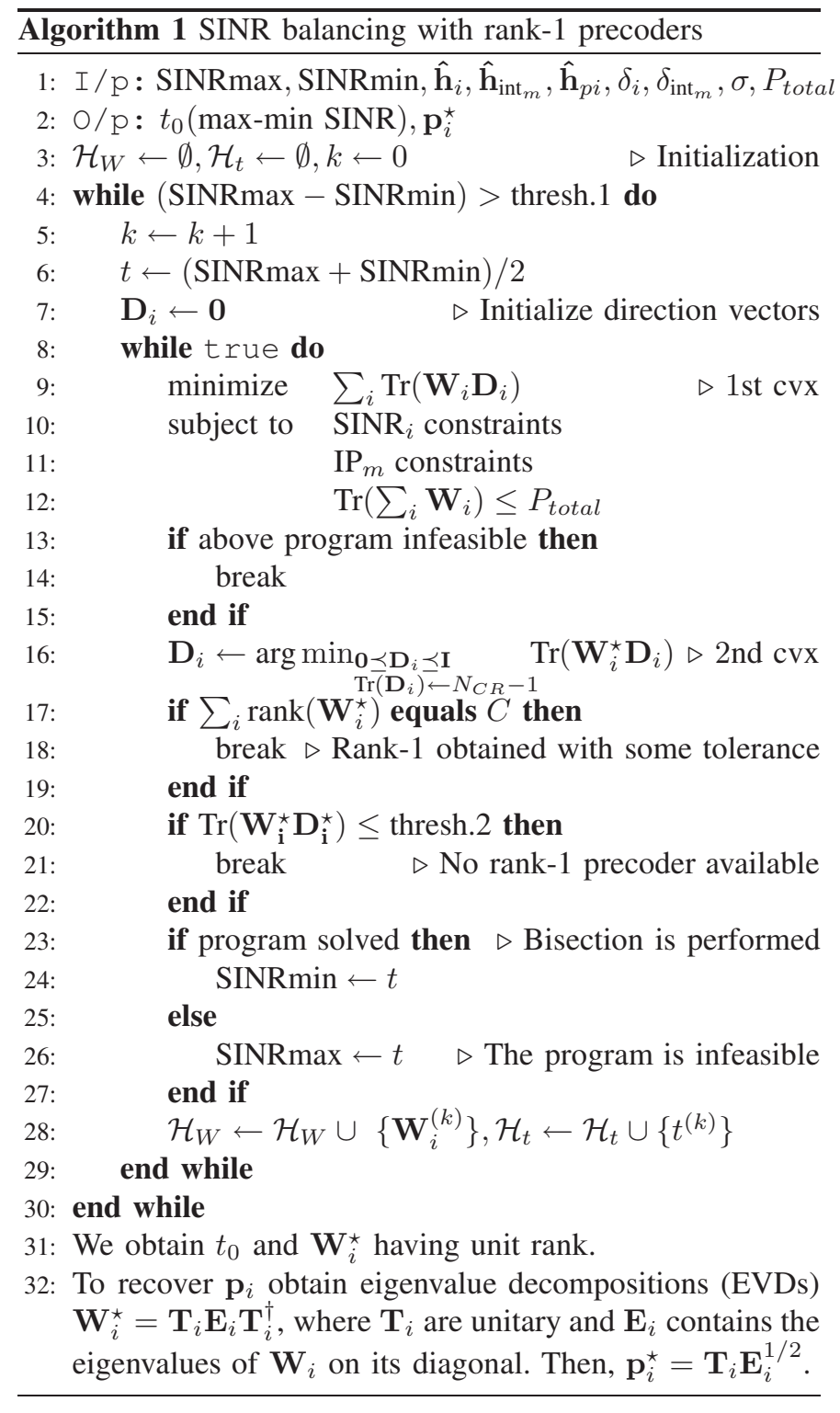

provides higher SINR, however, as seen in Fig. 1, such nonrobust beamformers perform poorly in terms of maintaining interference below the threshold at the PU RXs. For example, in this case the interference at PU RX 1 exceeded its threshold $64 \%$ of time.

Finally, in Fig. 3 we explore the effect of interference due to the presence of PU TXs. However, as mentioned above mathematical details supporting these results have been omitted due to space reasons. We assume there are 5 single antenna PU TXs and the interfering channel vector estimate experiences Rayleigh fading with corresponding error vector normally distributed, truncated to a sphere of radius $\delta$. In the "Std. Ray. Int." case the interfering signals are unit power and the powers are halved in the "Half power Int." case. Hence, the values of $P_{\text {total }} /\left(I+N_{0}\right)$ are $6.2 \mathrm{~dB}, 8.5 \mathrm{~dB}$ and 13.97 $\mathrm{dB}$ for the 3 curves in Fig. 3 where $I$ represents the total interference power. To model channel uncertainties we have taken $\epsilon=0.04, \delta=0.05$ in Fig. 3. It is seen that standard Rayleigh interference results in the worst performance. If the power of each of the interfering signals is halved, the mean rate of CR 1 increases from $2.45 \mathrm{bps} / \mathrm{Hz}$ to $3.07 \mathrm{bps} / \mathrm{Hz}$.
However, as is evident from Fig. 3, the best performance (mean rate of $4.5 \mathrm{bps} / \mathrm{Hz}$ ) is obtained in the absence of any such interference. This clearly shows that ignoring interference from the licensed users can drastically over estimate the quality-of-service expected to be provided by CR devices.

\section{CONCLUSION}

In this paper we have studied the the problem of maximizing the minimum SINR in the downlink of a multiple input single output CR network. The beamformers obtained also satisfy interference constraints at the PU RXs and total transmit power constraints at the CR BS. The optimal beamforming problem is tackled by exactly solving a SDP, to within a certain acceptable tolerance, without relaxing the rank-1 constraints. A low complexity algorithm implementing the proposed approach has been presented. Numerical simulations have been presented that show the effectiveness of the proposed procedure.

\section{REFERENCES}

[1] L. Zhang, R. Zhang, Y. C. Liang, Y. Xin, and H. V. Poor, "On Gaussian MIMO BC-MAC duality with multiple transmit covariance constraints." [Online]. Available: http://www.citebase.org/abstract?id=oai:arXiv.org: 0809.4101

[2] G. Zheng, K. K. Wong, and B. Ottersten, "Robust cognitive beamforming with bounded channel uncertainties," IEEE Transactions on Signal Processing, pp. 4871-4881, Dec. 2009.

[3] A. Beck and Y. Eldar, "Strong duality in nonconvex quadratic optimization with two quadratic constraints," SIAM J. Opt., vol. 17, no. 3, pp. 844-860, 2006.

[4] S. Boyd and L. Vandenberghe, Convex Optimization. Cambridge University Press, 2004.

[5] M. Bengtsson and B. Ottersten, "Optimal downlink beamforming using semidefinite optimization," in Proc. the 37th Annual Allerton Conf. Commun., Control and Comput., Illinois, USA, Sep. 1999, pp. 987-996.

[6] O. Filiz and A. Yener, "Rank constrained temporal-spatial matrix filters for CDMA systems," IEEE Transactions on Wireless Communications, vol. 3, no. 6, pp. 1974-1979, Nov. 2004.

[7] A. Wiesel, Y. C. Eldar, and S. Shamai, "Semidefinite relaxation for detection of 16-QAM signaling in MIMO channels," IEEE Signal Processing Letters, vol. 12, no. 9, pp. 653-656, Sep. 2005.

[8] W. K. Ma, T. N. Davidson, K. M. Wong, Z. Q. Luo, and P. C. Ching, "Quasi-maximum-likelihood multiuser detection using semi-definite relaxation with application to synchronous CDMA," IEEE Transactions on Signal Processing, vol. 50, no. 4, pp. 912-922, April 2002.

[9] Y. Huang and D. P. Palomar, "Rank-constrained separable semidefinite programming with applications to optimal beamforming," IEEE Transactions on Signal Processing, vol. 58, no. 2, pp. 664-678, Feb. 2010.

[10] G. Pataki, "On the rank of extreme matrices in semidefinite programs and the multiplicity of optimal eigenvalues," Mathematics of Operations Research, vol. 23, no. 2, pp. 339-358, 1998.

[11] J. Dattorro, Convex Optimization \& Euclidean Distance Geometry. Meboo Publishing USA, 2009.

[12] M. Botros and T. N. Davidson, "Convex conic formulations of robust downlink precoder designs with quality of service constraints," IEEE Journal of Selected Topics in Signal Processing, vol. 1, no. 4, pp. 714724, Dec. 2007.

[13] M. Schubert and H. Boche, "Solution of the multiuser downlink beamforming problem with individual SINR constraints," IEEE Transactions on Vehicular Technology, vol. 53, no. 1, pp. 18-28, Jan. 2004.

[14] A. Wiesel, Y. C. Eldar, and S. Shamai, "Linear precoding via conic optimization for fixed MIMO receivers," IEEE Transactions on Signal Processing, vol. 54, no. 1, pp. 161-176, Jan. 2006.

[15] A. Mutapcic, S. J. Kim, and S. Boyd, "A tractable method for robust downlink beamforming in wireless communications," in Proc. Asilomar Conference on Signals, Systems, and Computers (ACSSC), Asilomar, CA, Nov. 2007, pp. 1224-1228.

[16] M. Grant and S. Boyd, CVX: Matlab Software for Disciplined Convex Programming, June 2009. [Online]. Available: http://stanford. edu/ boyd/cvx 